\title{
A novel role of lamins from genetic disease to cancer biomarkers
}

\author{
Kunnathur Murugesan Sakthivel, ${ }^{1}$ Poonam Sehgal ${ }^{2}$ \\ ${ }^{1}$ Department of Biotechnology, Karunya University, Coimbatore, Tamilnadu, India; ${ }^{2}$ Chemical and \\ Biomolecular Engineering, University of Illinois, Urbana-Champaign, IL, USA
}

\begin{abstract}
Lamins are the key components of the nuclear lamina and by virtue of their interactions with chromatin and binding partners act as regulators of cell proliferation and differentiation. Of late, the diverse roles of lamins in cellular processes have made them the topic of intense debate for their role in cancer progression. The observations about aberrant localization or misexpression of the nuclear lamins in cancerous tissues have often led to the speculative role of lamins as a cancer risk biomarker. Here we discuss the involvement of lamins in several cancer subtypes and their potential role in predicting the tumor progression.
\end{abstract}

\section{Introduction}

The animal cell nuclei have a characteristic feature of a well-defined nuclear architecture and chromatin compartmentalization. The complex nuclear organization has been attributed to the increase in genome complexity and need for spatiotemporal regulation of the gene expression in higher vertebrates. Typical metazoan nucleus has been identified into three principal components- nuclear pore complex (NPC), nucleoplasm and lamina. The lamina is the meshwork of proteins found on the nucleoplasmic face of the inner nuclear membrane. A family of type $V$ intermediate filaments (IF) proteins called lamins is

Correspondence: Kunnathur Murugesan Sakthivel, Department of Biotechnology, Karunya University, Karunya Nagar, Coimbatore - 641114, Tamilnadu, India.

E-mail: sakthikm@gmail.com

Key words: Lamin A/C; lamin B; cancer; biomarker.

Acknowledgements: we greatly apologize to the Scientists, biologist, researcher and colleagues whose works were not cited due to space limitations. We are very thankful to Dr. Pankaj Chaturvedi for his useful comments on this manuscript.

Received for publication: 21 August 2016.

Revision received: 3 November 2016.

Accepted for publication: 4 November 2016.

This work is licensed under a Creative Commons Attribution NonCommercial 4.0 License (CC BY-NC 4.0).

(C) Copyright K.M. Sakthivel and P. Sehgal, 2016

Licensee PAGEPress, Italy

Oncology Reviews 2016; 10:309

doi:10.4081/oncol.2016.309 the principal component of this lamina. This family of proteins is found among all metazoans except Hydra and arthropods, and is localized exclusively in the nucleus unlike other members of intermediate filaments family. ${ }^{1}$ Absence of lamins or their homologs in plants and yeast support the notion that these proteins have evolved during the transition from open to closed mitosis. ${ }^{2}$ However, recent studies show that plants have a substitute for lamin proteins. Even though the lamin like proteins does not show sequence similarity, their secondary structure, nuclear distribution, their influence in nuclear shape and size suggests them as functional lamin analogs. ${ }^{3}$ In addition, homologs of metazoan lamins and lamin gene tree support the vertical evolution of lamin from the last eukaryotic common ancestor. ${ }^{4}$

As we move higher on the evolutionary scale, the number and complexity of lamin isoforms increases from single Imn-1 of C. elegans to two (Dm0 and lamC) in Drosophila to three (LMNB1, LMNB2, and $L M N A$ ) in human. ${ }^{5,6}$ The evolutionarily conserved lamins are subdivided into A- and B-type based on their biochemical properties, and for its conserved expression from worms to human, lamin B is considered as evolutionary precursor of lamin A. Among the lamin subtypes, B-type lamins have a ubiquitous expression and are considered essential for cell survival. A-type lamins, however, show a spatiotemporal expression during development and are majorly expressed in all differentiated cells and in some adult stem cells, while being considered absent in embryonic stem cells. ${ }^{7,8}$ Lamin A and lamin C (collectively referred as lamin $\mathrm{A} / \mathrm{C}$ ) are alternative splice variants of the $L M N A$ gene while lamin B1 and lamin B2 are transcribed from LMNB1 and LMNB2 respectively. Several studies have shown tissue-restricted expression of $L M N A$, minor splice variants lamin A 10, lamin C2, and also for lamin B3 a splice variant of $L M N B 2$, indicating the specialized roles of these proteins. ${ }^{9}$ Lamins $\mathrm{C} 2$ and $\mathrm{B} 3$ are shown to be germ-cell specific whereas lamin A 10 has been detected in cell lines derived from colon, lung, and breast carcinomas.

Lamins are known to be the major building blocks of nuclear structure, its shape, and provide mechanical steadiness to the cell nucleus by protecting them from mechanical forces especially in load bearing cells like muscles. They also directly or indirectly regulate gene expression, differentiation, DNA repair, and apoptosis. They bind to chromatin in a sequence independent manner or through their binding partners and are determining factors for chromatin positioning in the nucleus. ${ }^{10-12}$ Recently, disease causing A-type lamin mutants have been reported to be involved in regulating proteolytic degradation of proteins, affecting protein stability, and nuclear speckles ${ }^{13}$ stability. Lamins are known to interact with cytoskeleton through nuclear membrane SUN and KASH domain containing proteins and are crucial for mechanotransduction and mechanical stability of the cell. Studies with lamin $\mathrm{A} / \mathrm{C}$ deficient embryonic fibroblast have shown impaired mechanotransduction and decreased mechanical stiffness. ${ }^{14}$

The human $L M N A$ gene has 12 exons and studies spanning over two decades have reported more than 300 disease-causing mutations throughout the gene (9). These mutations lead to a wide variety of pleiotropic disorders with varying penetrance, which collectively with B-type lamin-associated diseases, are referred to as laminopathies. ${ }^{13}$ 
Depending on mutation involved, the laminopathies can affect a particular type of tissue or may manifest as a complex disorder affecting several tissues. The majority of the laminopathies affect tissues such as muscles, cardiomyocytes, adipocytes, and neurons, which are mesodermal in origin. Loss of binding of mutant lamin A/C to pRb, cyclin D3, and emerin has been attributed to defective myoblast differentiation due to reduction in MyoD, desmin, and M-cadherin, thereby leading to muscle degeneration/dystrophy. ${ }^{9}$ Two of the most studied laminopathies involving muscle tissues are Emery-Dreifuss muscular dystrophy (EMD) and limb-girdle muscular dystrophy (LGMD-1B). The late onset dilated cardiomyopathy and conduction-system disease (DCM-1A), the Charcot-Marie Tooth disorder, a neuropathy with peripheral nerve involvement (CMT-2B1), the Dunnigan-type familial partial lipodystrophy (FPLD), and the systemic disorders HutchinsonGilford progeria (HGPS), mandibuloacral dysplasia (MAD), and restrictive dermopathy (RD) have also been attributed to mutations in A-type lamins or their binding partners. On the other hand, mutations in Btype lamins are usually lethal and hence very rare. The only reported cases are of duplication of $L M N B 1$ leading to adult-onset autosomal dominant leukodystrophy (ADLD), a neurodegenerative disorder characterized by myelin loss in the central nervous system, ${ }^{15}$ and recently a $L M N B 1$ polymorphic variant was implicated as a modifier of neural tube closure defects. ${ }^{16}$ Individuals with $L M N B 2$ heterozygous mutations are found to be susceptible to acquired partial lipodystrophy. Despite extensive studies, a comprehensive explanation for tissue restricted phenotype and the mechanism is still elusive.

\section{Lamins and cancer}

Many researchers and cancer biologist very well ascribed the relationship between aberrant expression of lamin and cancer subtype by investigating the changes in expression profile of lamin in diverse types of cancers. The improper expression of lamins and its interaction with other proteins are often present in tumor cells. For example, Atype lamins interact with a number of transcription factors and regulates both differentiation and proliferation in cells. Lamin A/C binding regulates emerin, $\mathrm{pRb}, \mathrm{c}-\mathrm{Fos}$, SREBP1, MOK2 function and plays a role in p53, MAPK, ERK1/2, Wnt, TGF- $\beta$, Notch, and NF-k $\beta$ signaling. Studies with lamin A mutant overexpression have demonstrated a role for lamin A in myogenesis, adipogenesis, and osteogenesis. Expression of lamin A mutants in adult stem cells shows diminished potential to differentiate and regenerate tissues. Lamin $\mathrm{A}$ also regulates gene expression by binding chromatin to the nuclear periphery. Reduced or null expression of A-type lamins often correlates with low levels of differentiation and higher proliferation in cells. Furthermore, loss of lamin A leads to nuclear lobulations and changes in nuclear shape. ${ }^{17,18}$ Cancer cells are often characterized as highly proliferative with unregulated signaling, having irregular nuclear morphology and properties resembling stem cells..$^{19,20}$

The diverse functions and wide interactome of lamins have often led to the speculative role of lamins as a cancer risk biomarker that could predict the probability of tumor progression and therefore prognosis. In the following sections we emphasize the involvement of lamins in several cancers subtypes.

\section{Role of lamins in colorectal cancer}

Colorectal cancer is the third major type of cancer in both men and women not only in the United States but also worldwide. The aberrant or misexpression of lamins are also present in these type of cancers. A recent investigation provided a comprehensive link between lamin $\mathrm{A} / \mathrm{C}$ expression, patient prognosis and colorectal cancer (CRC) progression by comparing colorectal cancer and normal colon tissues for lamin expression. Using the Cox proportional hazard ratio (HR) method, patients were observed to have poor prognosis with almost two fold increase in mortality when the tumors tested positive for A-type lamin expression than patients with A-type lamin negative tumours. Lamin $\mathrm{A} / \mathrm{C}$ expression is majorly deficient from the cells of the colonic crypts except for a few basal crypt cells, which are believed to be stem cells. Ectopic expression of GFP-lamin A in colorectal cancer cells revealed increased in cell motility accompanied by an up-regulation of T-plastin, an actin bundling protein, and down regulation of E-cadherin, a protein involved in cell adherence. The study implicates lamin $\mathrm{A} / \mathrm{C}$ expression as a significant risk indicator of colorectal cancer -related mortality, probably due to increase in migratory and stem cell like properties. ${ }^{21}$

Another investigation revealed a correlation between A-type lamin expression and disease recurrence/clinical outcome of stage II and III colon cancer patients. Using paraffin embedded tissues and tissue microarrays, the authors observed low levels of lamin $\mathrm{A} / \mathrm{C}$ expression had a greater correlation with high disease recurrence, and suggested that these patients may benefit from adjuvant chemotherapy. ${ }^{22}$ They also observed that microsatellite stable tumors exhibited more frequently low level of LMNA expression than microsatellite instable tumors. Moreover, a recent study for role of a calcium binding protein S100A6 and its interacting protein $\beta$-catenin, a Wnt pathway effector, in colorectal cancer tissues found that high levels of S100A6 expression is observed in metastatic versus non-metastatic human colorectal cancer cell lines. S100A6 protein was also established as a novel interacting partner of lamin $\mathrm{A} / \mathrm{C}$ protein, hence potentially linking lamin $\mathrm{A} / \mathrm{C}$ in colorectal cancer development and progression. ${ }^{23}$ This clearly indicates that improper regulation of lamins leads to various type of gastrointestinal cancers, in later section of the review we shall discuss other form of gastrointestinal cancers also.

\section{Role of lamins in pancreatic cancer}

A recent study designed to look at the mechanism of betulinic acid treatment of pancreatic cancer discovered lamin B1 overexpression in pancreatic cancer. The drug betulinic acid shows antitumor property by down-regulating lamin B1 expression. Lamin B1 overexpression could serve as a biomarker in pancreatic cancer as the study found it to be associated with more malignant form of cancer with poor prognosis of patients. $^{24}$

\section{Role of lamins in other gastrointestinal cancer}

The various intermediate pathological steps of gastrointestinal cancer are easily identifiable, making it easier to observe the changes in expression of nuclear lamins accompanying cancer progression. ${ }^{25}$ But, there have been a few studies linking expression of nuclear lamins and progression of gastrointestinal cancer. In one such study it was found that in gastrointestinal neoplasm both types of lamins have reduced expression with A-type lamins having a more pronounced effect in case of gastric dysplasia. However, no such reduction was observed in stages of intestinal metaplasia and gastric atrophy. This reduction in expression was accompanied by aberrant, cytoplasmic detection of lamins by immunolabelling. Comparative studies with other solid state tumors showed reduced expression of both lamins- $\mathrm{A} / \mathrm{C}$ and $\mathrm{B} 1$, frequently in squamous and adenocarcinoma of the esophagus, cervical and uterine cancers, breast cancer, and bronchial carcinoma but not in pancreatic 
and hepatic cancer. Hence, reduced expression of nuclear lamins may serve as a potential indicator of early stages of gastrointestinal cancer and cytoplasmic detection as an indicator of a more malignant form. ${ }^{26}$

\section{Role of lamins in neuroblastoma}

Lamin A/C shows spatiotemporal expression during development and has a potential role in neurogenesis. ${ }^{8}$ Neuroblastoma is a solid tumor frequently observed in childhood involving primitive cells of the sympathetic nervous system with an ability to undergo differentiation. A key therapeutic intervention for this aggressive tumor is to induce differentiation by various chemical agents. ${ }^{27}$ The observation that initial stages of human neuroblastomas show reduced expression of lamin $\mathrm{A} / \mathrm{C}$ protein in the majority of these cases prompted to study the role of A-type lamins in differentiation and progression of neuroblastoma by knocking down lamin $\mathrm{A} / \mathrm{C}$ in neuroblastoma cells. Cells with depleted lamin A/C levels fail to undergo retinoic acid-induced differentiation have increased cell migration and drug resistance. The inability to differentiate is further indicated by the absence of distinctive neurites outgrowth and reduced expression of neural markers. ${ }^{28}$ Collectively, the studies indicate that reduced levels of lamin $\mathrm{A} / \mathrm{C}$ expression could be used as a diagnostic tool for the more aggressive form of neuroblastoma.

\section{Role of lamins in prostate cancer}

A study on progression of various states of prostate cancer by comparing several human prostate cancer cell lines observed a correlation between post-translational modification of B-type lamin and the state of differentiation/proliferation in prostate cancer cells. Although the expression levels of B-type lamins were comparable between the cell lines, the malignant PC3 cells showed an increase in lamin B phosphorylation. As the lamins have been identified as a primary component of Nuclear matrix (NM) and Matrix Associated Regions (MARs) the authors speculate that modifying the interactions between NM and MARs may affect gene expression giving rise to a more malignant phenotype. ${ }^{29}$ These results are supported by an independent study by a different group which reported that knock-down of a nuclear protein MeCP2 in PC3 and LNCaP cells causes aberrant proliferation and defective cell cycle progression. This defect is accompanied by diminished lamin $\mathrm{A} / \mathrm{C}$, lamin $\mathrm{B} 1$ and lamin $\mathrm{B}$ receptor (LBR) protein levels and altered nuclear shape. ${ }^{30}$ Since, MeCP2 interacts with LBR and HP1 to anchor chromatin at the nuclear periphery, its deficit might lead to reduced cell proliferation and viability. Another study involving prostate cancer cell lines observed increase in lamin B-deficient microdomains (LDMDs) and nuclear lobulation, often correlating with augmented aggressiveness and motility of prostate cancer cells. Genes localizing to LDMDs show decreased expression due to stalled Pol II at the promoters in that region. The authors demonstrated that chromosomal regions linked to prostate cancer susceptibility mostly localize to LDMDs. ${ }^{31}$ These observations provide mechanistic insights of B-type lamins in development and progression of prostate cancer.

In another investigation using different prostate cancer cell lines as model for disease progression, found that increased A-type lamin expression leads to increased cell growth, colony formation, and malignancy of prostate cancer cells. It can be argued that increased A typelamin expression may modulate PI3K/AKT/PTEN signaling pathway by altered mechanotransduction between and nucleus and cytoplasmic membrane and lead to aberrant cell proliferation..$^{32}$ By using 2D-DIGE and MALDI-TOF/TOF mass spectrometry Skvortsov et al. reported lamin
A is a differentially expressed abundant protein between low and high Gleason score prostate tumors. These observations clearly put forward that A type-lamins might serve as a biomarker of tumor differentiation and prognosis and as a novel therapeutic target for prostate cancer. ${ }^{33}$

\section{Role of lamins in germ cell cancer}

Seminoma and non-seminoma are two main types of germ cell tumors that occur in men. They differ in growth rate with non-seminoma having more rapid growth in comparison. Most non-seminoma tumors are of mixed type and the percentage of embryonic carcinoma subtype predicts the malignancy of germ cell tumor. ${ }^{34}$ In an attempt to study lamin expression in testicular germ cell tumors, cryo-preserved tissue sections of the normal testis and various other testicular germ cell tumors were coimmunostained for both A- and B-type lamins. In testicular germ cell tumors, while B-type lamins were frequently found to be expressed, A-type lamins depicted differential expression with only lamin $\mathrm{C}$ being expressed in embryonic carcinoma. This differential expression could help establish detection of embryonic carcinoma in tumors and act as a prognostic marker. ${ }^{35}$

\section{Role of lamins in liver cancer}

Like other cancers Hepatocellular carcinoma (HCC) also involves aberrant gene expression and is frequently observed along with liver cirrhosis. Lamin B1 may be considered as a marker for cirrhosis, because its expression level changes considerably in cirrhotic tissue as compared to normal tissue. ${ }^{36}$ The expression level of not only Lamin B1 but also both the subtypes of lamins was investigated in hepatocellular regeneration during liver cirrhosis and in different grades of hepatocellular carcinomas. Immunohistochemistry on frozen tissue sections revealed lamin expression both in cirrhosis and carcinomas. ${ }^{37}$ The proteomic expression profiling and clinicopathological study of diseasefree and patients suffering from cirrhotic liver and HCC identified lamin B1 and vimentin as predominant proteins elevated in cancerous tissues. Circulating lamin B1 and vimentin could serve as novel biomarkers of early stage HCC that could be detected by noninvasive method. ${ }^{38}$ Another group performed similar proteomic analysis of normal and cancerous tissue and identified sarcosine dehydrogenase, liver carboxylesterase, peptidyl-prolyl isomerase A, and lamin B1 as novel hepatocellular carcinoma biomarkers.$^{36}$ Hence, testing for increase in lamin B1 expression could lead to early detection of hepatocellular carcinoma. Thus, Lamin in combination with other liver enzymes that overexpressed in Hepatocellular carcinoma could be a good diagnostic marker.

\section{Role of lamins in lung cancer}

The correlation between lamin expression and lung cancer was one of the earliest investigation relating lamins to cancer progression..$^{38}$ Kaufmann group has shown that the A-type lamins decreased in small cell lung cancer (SCLC) cell lines. They also demonstrated that lamin $\mathrm{A} / \mathrm{C}$ levels were more than $80 \%$ lower in SCLC cell lines compared to non-SCLC lines. ${ }^{38,39}$ When lamin expression was compared between small cell lung cancer (SCLC), squamous cell carcinomas, and adenocarcinomas it was found that lamin B expression was unaltered, and lamin A/C expression was weaker in SCLC cell lines compared to nonSCLC cell lines. ${ }^{39}$ Similar results were observed when A and B- type 
lamin expression was compared between various non-SCLC and SCLC lines. Expression of v-rasH oncogene in the NCI-H249 small cell line gives rise to phenotype resembling large cell carcinoma of the lung and increase in expression of lamin $\mathrm{A} / \mathrm{C}$ and vimentin. Here, increase in lamin $\mathrm{A} / \mathrm{C}$ expression is associated with increased malignancy of lung cancer. ${ }^{40}$ Another study took a closer look at the protein expression profile of cancer cell line A549 and compared it to normal lung fibroblast cell line MRC-5. Lamin A/C was found to be overexpressed in A549 cells and was postulated to be a biomarker of lung cancer for early detection. ${ }^{41}$ Studying the expression of A-type lamins in lung adenocarcinoma cell line GLC-A1 showed that not only was the expression of lamin $\mathrm{A} / \mathrm{C}$ reduced, but also there were significantly higher levels of lamin C compared to lamin A. ${ }^{42}$ Recent studies with A549 cell lines and green tea polyphenols reported to have antitumor properties showed that green tea extract induced upregulation of lamin $\mathrm{A} / \mathrm{C}$ expression. The increased A-type lamins in turn can lead to altered actin remodeling and consequently, reduced cell motility. The result is in contradiction to earlier discussed report as authors here show that lamin $\mathrm{A} / \mathrm{C}$ overexpression seemingly reduces the migratory property of lung cancer cells. ${ }^{43}$ In most of the lung cancer, B-type lamins are generally overexpressed. Taken together, we can clearly see that Lamin A/C act as diagnostic marker in early stages of lung cancer while Lamin B1 can be a good marker for later stages of cancer.

\section{Role of lamins in skin cancer}

Expression patterns of lamin subtypes between normal human skin, actinic keratosis, squamous cell carcinoma (SSC) and basal cell carcinoma (BCC) were correlated to their proliferative potential using immunohistochemistry. Though A and B-type lamins are expressed in both normal and cancerous epidermal cells, a high percentage of proliferating cells found in basal and squamous cell carcinomas stain positive for lamin A expression, suggesting these cells may undergo differentiation. ${ }^{44}$ Another important oncogene that correlates well with BCC progression is GLI1. A small molecule inhibitor of the hedgehog signaling, Vismodegib has been recently approved by FDA for BCC treatment. While many strategies have been documented to overcome GLIl's role in cancer, ${ }^{20,45}$ especially BCC, ${ }^{46}$ the relationship between lamins and GLI1 has to be elucidated in detail. Another study examined the importance of variation in lamin expression as a diagnostic marker in keratinocytic tumors. The expression of all kinds of lamins was reduced with lamin B showing heterogenous pattern in differentiated SCCs and keratoacanthomas. ${ }^{47} \mathrm{~A}$ detailed analysis of lamin subtypes was performed in tissue sections of basal cell carcinomas leading to the categorization of four types of cells lamin A negative, lamin $\mathrm{C}$ negative, lamin $\mathrm{A} / \mathrm{C}$ negative and lamin A/B2 negative. Correlation of these cell subtypes to proliferation rates revealed that absence of lamin A is associated with high proliferation rates and absence of lamin $\mathrm{C}$ with slow growth rate, hence implicating absence of A-type lamin expression in cancer progression. ${ }^{48}$ A proteomic profile between normal human oral keratinocytes and oral squamous cell carcinomas derived cell lines found that twenty-two proteins were differentially expressed including proteins like annexin A1, heat shock protein 27 and lamin A/C. ${ }^{49}$ These investigations explore a possible avenue for A-type lamins as a signature molecule for oral cancer diagnosis.

\section{Role of lamins in ovarian cancer}

Recently, in a proteomic exploration to identify potential biomarkers for ovarian cancer (OC) in women with polycystic ovary syndrome
(PCOS), tissue samples from women with and without OC were compared. The authors found that six biomarkers calreticulin, fibrinogen$\gamma$, superoxide dismutase, vimentin, malate dehydrogenase, and lamin $\mathrm{B} 2$ were overexpressed both in women with $\mathrm{OC}$ and in women with PCOS. These biomarkers could help identify the possible risk of ovarian cancer in women with PCOS. ${ }^{50}$ Further studies would be necessary to evaluate the true potential of these biomarkers.

\section{Role of lamins in breast cancer}

Samples of breast cancer and associated non-cancerous tissues were examined to correlate the expressions of lamin A/C, lamin Bland LBR to various stages of human breast cancer and their clinical outcome. While higher expression of $L M N A$ were associated with early stages of cancer and hence favorable prognosis, the expression of $L M N B 1$ correlated directly to the tumor grade and declined with increasing probability of mortality. Hence, in breast cancer, the decreased expression of $L M N B 1$ associates with poor prognosis. ${ }^{51}$ In another study, null expression of A-type lamins in a majority of cancerous tissue or aberrant, heterogeneous expression in breast cancer cells was reported. Knockdown of lamin $\mathrm{A} / \mathrm{C}$ expression by shRNA led to cancer like altered morphology and aneuploidy in primary breast epithelial cells, implicating A-type lamins in breast cancer progression. ${ }^{2}$

\section{Role of lamins in progeria disorder}

Progeria or premature aging is a severe systemic disorder caused by mutations that lead to altered lamin A processing that leads to formation of a truncated protein progerin. Progerin also accumulates in different tissues of normal individuals as they age, suggesting it has a role in normal aging. ${ }^{53}$ Aging also leads to genomic instability, which is one of the leading risk factor for cancers, raising speculations about correlation between progerin and cancer. Mouse models in which cells accumulate prelamin A could help establish the relationship between prelamin A accumulation and invasive properties of cancer. Silencing of gene ZMPSTE24 in breast, oral, and lung cancer model causes progerin accumulation and changes in proteoglycan synthesis pathway leading to increased production of over-sulfated forms of chondroitin sulfate and heparan sulfate. These changes in the ECM components could lead to reduced invasive potential and establish progerin as a safeguard against cancer. Experiments performed in mosaic mouse models comprising of both prelamin A and normal mature lamin A expressing cells indicate that prelamin A does not affect tumor initiation, but suggests that it prevents cancer invasion. ${ }^{54}$ Another study investigating the role of progerin in human prostate, breast and colon cancer cell lines detected higher than normal expression of progerin in cancer cells. Ectopic progerin expression does not cause cellular senescence in cancer cells, and this could be attributed to defective DNA damage repair associated with progeria. Based on these results the authors hypothesize that progerin could promote tumor formation by increasing either DNA damage or genomic instability. ${ }^{55}$ However, no thorough clinical investigation to corroborate progerin's role in cancer has been made, possibly due to quite a short lifespan of progeria patients.

\section{Lamins-prognostic or diagnostic biomarker?}

Cell motality, cell migration, and invasion are one of the critical fac- 
Table 1. Role of different types of lamins in various types of cancers.

\begin{tabular}{llll} 
Type of cancer & Type of lamins involved & Phenotype of lamin & Changes in nuclear shape/sive \\
Hepatocellular carcinoma & Lamin B & $\begin{array}{l}\text { Increased expression of LaminB } \\
\text { Increased expression of Lamin B in Plasma }\end{array}$ & Enlargement of nucleus \\
Prostate cancer & Lamin & Increased expressionof lamin B & Enlargement of nucleus \\
\hline BCC & LaminA/C & Low expression of LaminA/C & Enlargement of nucleus \\
Colon cancer (Later stages) & LaminA/C & Low expression of LaminA/C & Enlargement of nucleus \\
\hline Gastrointestinal Neoplasms & LaminA/C & Aberrant or decreased LaminA/C expression & Enlargement of nucleus \\
Gastric carcinoma & LaminA/C & Decreased LaminA/C mRNA and protein expression & Enlargement of nucleus \\
\hline Breast cancer & LaminA/C & Decreased LaminA/C expression & Enlargement of nucleus \\
Neuroblastoma & LaminA/C & Decreased LaminA/C expression & Enlargement of nucleus \\
\hline
\end{tabular}

tors during the progression of metastasis. Various studies have already indicated that undeniably lamin $\mathrm{A} / \mathrm{C}$ is involved in cell proliferation, migration and invasion of various cancer cells. Enlargement and distortion of nuclear shape are characteristic features of a malignant cell also. As lamins play a vital role in providing structural and mechanical strength to the nucleus, their role in cancer has been under considerable scrutiny. Lamins often show aberrant expression and localization in cancer cells as shown in Table 1. Aberrant localization of lamin $\mathrm{A} / \mathrm{C}$ to cytoplasm has been observed in various cancers like lung, colorectal and gastric cancers. ${ }^{21,26,39}$

Lamins are also shown to regulate oxidative stress in the cancer cells. ${ }^{56}$ While the low level ROS induces cell proliferation, increased ROS often results in DNA damage. ROS also acts as a basic signaling molecule in both the normal and cancer cells. Radiation or chemotherapeutic agents induced bystander effect is also mediated mostly by ROS. ${ }^{57-60}$ However, the role of lamin in cancer cell and bystander cells has to be elucidated in detail. The regulation of DNA damage and repair proteins, especially DNA double strand break repair proteins like ATM, ATR, H2AX, ${ }^{61}$ BRCA1, FANCD2 ${ }^{62-64}$ has to be tightly regulated to avoid the genomic instability and carcinogenesis. The dysregulation of A-type lamins impacts transcription, DNA replication and repair, and epigenetic modification of chromatin, hence inducing genomic instability that can contribute to cancer progression. ${ }^{65}$ Thus, it will be quite interesting to study the nuclear and cytoplasmic role of lamins in cancer progression and its recurrence. Role of lamins in both promotion and inhibition of apoptosis suggests a strong correlation between expression of lamins and malignancy of tumor cells. ${ }^{66}$

The current ambiguity in assigning lamins as a cancer biomarker is due to its variable expression between cancer subtypes. ${ }^{67}$ It is well known that Lamin B is universally expressed in many cell types, even cancer cells, making them a poor diagnostic marker for most studies except in the case of HCC where its overexpression is observed in both early and late stages of cancer. Similarly, lamin A/C might not serve as a useful diagnostic biomarker due to the variability between cancer subtypes. Larger statistical studies are necessary before clinical diagnosis utilizes aberrant expression or localization of lamins as a diagnostic biomarker for cancer.

Differentiation of tumor cells correlates with cancer prognosis, with higher differentiation correlating with better prognosis and low differentiation leading to poor prognosis. ${ }^{67}$ Lamin B potential to become a prognosis marker has been well studied in case of prostate and pancreatic cancer where it is associated with the more malignant form of cancer. Expression of lamin A/C is often used to demarcate differentiated cancer cells. Lamin A/C expression is also shown to alter expression of E-cadherin, which leads to reduced cell adhesion. Increase in cancer cell motility leads to metastasis and hence significantly worsens the prognosis. ${ }^{67}$ Therefore, based on the subtype of cancer involved lamin $\mathrm{A} / \mathrm{C}$ has the potential to be a cancer biomarker, but detailed mechanis- tic investigations for the role of lamins in cancers are still wanting. This also indicates that lamins can be also used as prognostic marker in combination with other cancer markers. The role of Progerin in development of cancer is also not well elucidated and this could be another important area where we have to emphasize more. It will be also interesting to study the role of other nuclear lamin related protein like emerin and LAP2 alpha (Lamina-associated polypeptide 2 ) which also overecpressed in various cancer cells. The cross talk between these proteins and lamins are not so well studied.

\section{Conclusions}

In conclusion, lamin is over expressed in most of the cancers and has the ability to maintain the cancer cells homeostasis. Especially, lamin maintains the cell differentiation, proliferation and motility in tumors, which is essential for aggressive tumors. Conversely, contradicting results exist regarding role of lamins in cancer. This ambiguity could be answered by considering the numerous diverse functions that lamins perform in the cell and to relate its expression pattern in context to its role in cancers that arise from different cell types. As highlighted in the present review, lamin can be a potential diagnostic biomarker compared to prognostic marker. However, 'bench to bedside' approach to correlate large number of clinical samples and lamin expression analysis in these clinical samples can provide a better insight for cancer diagnostics.

\section{References}

1. Szeverenyi I, Cassidy AJ, Chung CW, et al. The Human Intermediate Filament Database: comprehensive information on a gene family involved in many human diseases. Hum Mutat 2008;29:351-60.

2. Goldman RD, Goldman AE, Shumaker DK. Nuclear lamins: building blocks of nuclear structure and function. Novartis Found Symp 2005;264:227-30.

3. Ciska M, Moreno Díaz de la Espina S. The intriguing plant nuclear lamina. Front Plant Sci 2014;5:166.

4. Koreny L, Field MC. Ancient eukaryotic origin and evolutionary plasticity of nuclear lamina. Genome Biol Evol 2016;8:2663-71.

5. Hutchison CJ. Lamins: building blocks or regulators of gene expression? Nat Rev Mol Cell Biol 2002;3:848-58.

6. Lin F, Worman HJ. Structural organization of the human gene encoding nuclear lamin A and nuclear lamin C. J Biol Chem 1993;268:16321-6. 
7. Constantinescu D, Gray HL, Sammak PJ, et al. Lamin A/C expression is a marker of mouse and human embryonic stem cell differentiation. Stem Cells Dayt Ohio 2006;24:177-85.

8. Sehgal P, Chaturvedi P, Kumaran RI, et al. Lamin A/C haploinsufficiency modulates the differentiation potential of mouse embryonic stem cells. PloS One 2013;8:e57891.

9. Parnaik VK. Role of nuclear lamins in nuclear organization, cellular signaling, and inherited diseases. Int Rev Cell Mol Biol 2008;266:157-206.

10. Stierlé V, Couprie J, Ostlund C, et al. The carboxyl-terminal region common to lamins $\mathrm{A}$ and $\mathrm{C}$ contains a DNA binding domain. Biochemistry (Mosc) 2003;42:4819-28.

11. Vlcek S, Foisner R. A-type lamin networks in light of laminopathic diseases. Biochim Biophys Acta 2007;1773:661-74.

12. Chaturvedi P, Parnaik VK. Lamin A rod domain mutants target heterochromatin protein 1alpha and beta for proteasomal degradation by activation of F-box protein, FBXW10. PLoS One 2010;5:e10620.

13. Tripathi K, Muralukrishna B, Parnaik VK. Differential dynamics and stability of lamin A rod domain mutants. Int $\mathrm{J}$ Integr Biol 2009;5:1-8.

14. Lammerding J, Schulze PC, Takahashi T, et al. Lamin A/C deficiency causes defective nuclear mechanics and mechanotransduction. J Clin Invest 2004;113:370-8.

15. Padiath QS, Saigoh K, Schiffmann R, et al. Lamin B1 duplications cause autosomal dominant leukodystrophy. Nat Genet 2006;38:1114-23.

16. De Castro SCP, Malhas A, Leung K-Y, et al. Lamin b1 polymorphism influences morphology of the nuclear envelope, cell cycle progression, and risk of neural tube defects in mice. PLoS Genet 2012;8:e1003059.

17. Shimi T, Pfleghaar K, Kojima S, et al. The A- and B-type nuclear lamin networks: microdomains involved in chromatin organization and transcription. Genes Dev 2008;22:3409-21.

18. Burke B, Stewart CL. The nuclear lamins: flexibility in function. Nat Rev Mol Cell Biol 2013;14:13-24.

19. Meng E, Mitra A, Tripathi K, et al. ALDH1A1 maintains ovarian cancer stem cell-like properties by altered regulation of cell cycle checkpoint and DNA repair network signaling. PLoS One 2014;9:e107142.

20. Tripathi K, Mani C, Barnett R, et al. Gli1 Regulates S-phase Checkpoint in Tumor Cells via Bid and its Inhibition Sensitizes to DNA Topoisomerase 1 Inhibitors. J Biol Chem 2014;24.

21. Willis ND, Cox TR, Rahman-Casañs SF, et al. Lamin A/C is a risk biomarker in colorectal cancer. PLoS One 2008;3:e2988.

22. Belt EJT, Fijneman RJA, van den Berg EG, et al. Loss of lamin A/C expression in stage II and III colon cancer is associated with disease recurrence. Eur J Cancer Oxf Engl 1990 2011;47:1837-45.

23. Kila czyk E, Graczyk A, Ostrowska H, et al. S100A6 is transcriptionally regulated by $\beta$-catenin and interacts with a novel target, lamin A/C, in colorectal cancer cells. Cell Calcium 2012;51:470-7.

24. Li L, Du Y, Kong X, et al. Lamin B1 is a novel therapeutic target of betulinic acid in pancreatic cancer. Clin Cancer Res Off J Am Assoc Cancer Res 2013;19:4651-61.

25. Correa P. Human gastric carcinogenesis: a multistep and multifactorial process-First American Cancer Society Award Lecture on Cancer Epidemiology and Prevention. Cancer Res 1992;52:6735-40.

26. Moss SF, Krivosheyev V, de Souza A, et al. Decreased and aberrant nuclear lamin expression in gastrointestinal tract neoplasms. Gut 1999;45:723-9.

27. Brodeur GM. Neuroblastoma: biological insights into a clinical enigma. Nat Rev Cancer 2003;3:203-16.

28. Maresca G, Natoli M, Nardella M, et al. LMNA knock-down affects differentiation and progression of human neuroblastoma cells. PLoS One 2012;7:e45513.
29. Barboro P, Repaci E, D’Arrigo C, Balbi C. The role of nuclear matrix proteins binding to matrix attachment regions (Mars) in prostate cancer cell differentiation. PLoS One 2012;7:e40617.

30. Babbio F, Castiglioni I, Cassina C, et al. Knock-down of methyl CpGbinding protein 2 (MeCP2) causes alterations in cell proliferation and nuclear lamins expression in mammalian cells. BMC Cell Biol 2012;13:19.

31. Helfand BT, Wang Y, Pfleghaar K, et al. Chromosomal regions associated with prostate cancer risk localize to lamin B-deficient microdomains and exhibit reduced gene transcription. J Pathol 2012;226:735-45.

32. Kong L, Schäfer G, Bu H, et al. Lamin A/C protein is overexpressed in tissue-invading prostate cancer and promotes prostate cancer cell growth, migration and invasion through the PI3K/AKT/PTEN pathway. Carcinogenesis 2012;33:751-9.

33. Skvortsov S, Schäfer G, Stasyk T, et al. Proteomics profiling of microdissected low- and high-grade prostate tumors identifies Lamin A as a discriminatory biomarker. J Proteome Res 2011;10:259-68.

34. Ulbright TM. Germ cell tumors of the gonads: a selective review emphasizing problems in differential diagnosis, newly appreciated, and controversial issues. Mod Pathol Off J U S Can Acad Pathol 2005;18:S61-79.

35. Machiels BM, Ramaekers FC, Kuijpers HJ, et al. Nuclear lamin expression in normal testis and testicular germ cell tumours of adolescents and adults. J Pathol 1997;182:197-204.

36. Lim SO, Park S-J, Kim W, et al. Proteome analysis of hepatocellular carcinoma. Biochem Biophys Res Commun 2002;291:1031-7.

37. Hytiroglou P, Choi SW, Theise ND, et al. The expression of nuclear lamins in human liver: an immunohistochemical study. Hum Pathol 1993;24:169-72.

38. Wong K-F, Luk JM. Discovery of lamin B1 and vimentin as circulating biomarkers for early hepatocellular carcinoma. Methods Mol Biol Clifton NJ 2012;909:295-310.

39. Broers JL, Raymond Y, Rot MK, et al. Nuclear A-type lamins are differentially expressed in human lung cancer subtypes. Am J Pathol 1993;143:211-20.

40. Kaufmann SH, Mabry M, Jasti R, Shaper JH. Differential expression of nuclear envelope lamins $\mathrm{A}$ and $\mathrm{C}$ in human lung cancer cell lines. Cancer Res 1991;51:581-6.

41. Rubporn A, Srisomsap C, Subhasitanont P, et al. Comparative proteomic analysis of lung cancer cell line and lung fibroblast cell line. Cancer Genom Proteom 2009;6:229-37.

42. Machiels BM, Broers JL, Raymond Y, et al. Abnormal A-type lamin organization in a human lung carcinoma cell line. Eur J Cell Biol 1995;67:328-35.

43. Lu Q-Y, Yang Y, Jin YS, et al. Effects of green tea extract on lung cancer A549 cells: proteomic identification of proteins associated with cell migration. Proteomics 2009;9:757-67.

44. Tilli CMLJ, Ramaekers FCS, Broers JLV, et al. Lamin expression in normal human skin, actinic keratosis, squamous cell carcinoma and basal cell carcinoma. Br J Dermatol 2003;148:102-9.

45. Palle K, Mani C, Tripathi K, Athar M. Aberrant GLI1 Activation in DNA damage response, carcinogenesis and chemoresistance. Cancers 2015;7:2330-51.

46. Athar M, Tang X, Lee JL, et al. Hedgehog signalling in skin development and cancer. Exp Dermatol 2006;15:667-77.

47. Oguchi M, Sagara J, Matsumoto K, et al. Expression of lamins depends on epidermal differentiation and transformation. Br J Dermatol 2002;147:853-8.

48. Venables RS, McLean S, Luny D, et al. Expression of individual lamins in basal cell carcinomas of the skin. Br $\mathrm{J}$ Cancer 2001;84:512-9.

49. Hudson ME, Pozdnyakova I, Haines K, et al. Identification of differ- 
entially expressed proteins in ovarian cancer using high-density protein microarrays. Proc Natl Acad Sci U S A 2007;104:17494-9.

50. Galazis N, Olaleye 0, Haoula Z, et al. Proteomic biomarkers for ovarian cancer risk in women with polycystic ovary syndrome: a systematic review and biomarker database integration. Fertil Steril 2012;98:1590-1601.e1.

51. Wazir U, Ahmed MH, Bridger JM, et al. The clinicopathological significance of lamin $\mathrm{A} / \mathrm{C}$, lamin $\mathrm{B} 1$ and lamin $\mathrm{B}$ receptor mRNA expression in human breast cancer. Cell Mol Biol Lett 2013;18:595611.

52. Capo-chichi CD, Cai KQ, Smedberg J, et al. Loss of A-type lamin expression compromises nuclear envelope integrity in breast cancer. Chin J Cancer 2011;30:415-25.

53. Butin-Israeli V, Adam SA, Goldman AE, Goldman RD. Nuclear lamin functions and disease. Trends Genet TIG 2012;28:464-71.

54. de la Rosa J, Freije JMP, Cabanillas R, et al. Prelamin A causes progeria through cell-extrinsic mechanisms and prevents cancer invasion. Nat Commun 2013;4:2268.

55. Tang Y, Chen Y, Jiang H, Nie D. Promotion of tumor development in prostate cancer by progerin. Cancer Cell Int 2010;10:47.

56. Shimi T, Goldman RD. Nuclear lamins and oxidative stress in cell proliferation and longevity. Adv Exp Med Biol 2014;773:415-30.

57. Chinnadurai M, Chidambaram S, Ganesan V, et al. Bleomycin, neocarzinostatin and ionising radiation-induced bystander effects in normal diploid human lung fibroblasts, bone marrow mesenchymal stem cells, lung adenocarcinoma cells and peripheral blood lymphocytes. Int J Radiat Biol 2011;87:673-82.

58. Chinnadurai M, Paul SFD, Venkatachalam P. The effect of growth architecture on the induction and decay of bleomycin and X-rayinduced bystander response and genomic instability in lung adenocarcinoma cells and blood lymphocytes. Int $\mathrm{J}$ Radiat Biol
2013;89:69-78.

59. Chinnadurai M, Rao BS, Deepika R, et al. Role of reactive oxygen species and nitric oxide in mediating chemotherapeutic drug induced bystander response in human cancer cells exposed invitro. World J Oncol 2012;3:64-72.

60. Basheerudeen SAS, Mani C, Kulkarni MAK, et al. Human brain glioblastoma cells do not induce but do respond to the bleomycininduced bystander response from lung adenocarcinoma cells. Mutat Res 2013;757:114-9.

61. Rakiman I, Chinnadurai M, Baraneedharan U, et al. $\gamma$-H2AX assay: a technique to quantify DNA double strand breaks. Adv Biotech 2008;39-41.

62. Tripathi K, Mani C, Clark DW, Palle K. Rad18 is required for functional interactions between FANCD2, BRCA2, and Rad51 to repair DNA topoisomerase 1-poisons induced lesions and promote fork recovery. Oncotarget 2016;7:12537-53.

63. Clark DW, Tripathi K, Dorsman JC, Palle K. FANCJ protein is important for the stability of FANCD2/FANCI proteins and protects them from proteasome and caspase-3 dependent degradation. Oncotarget 2015;6:28816-32.

64. Tripathi K, Mani C, Somasagara RR, et al. Detection and evaluation of estrogen DNA-adducts and their carcinogenic effects in cultured human cells using biotinylated estradiol. Mol Carcinog 2016 [Ahead of print].

65. Gonzalo S. DNA damage and lamins. Adv Exp Med Biol 2014;773:377-99.

66 . Broers JLV, Ramaekers FCS. The role of the nuclear lamina in cancer and apoptosis. Adv Exp Med Biol 2014;773:27-48.

67. Foster CR, Przyborski SA, Wilson RG, Hutchison CJ. Lamins as cancer biomarkers. Biochem Soc Trans 2010;38:297-300. 